\title{
HYBRID ORGANIC-INORGANIC COMPOSITE MATERIALS FOR APPLICATION IN CHEMICAL SENSORS
}

\author{
Anca-Dana Bendrea*, Ana-Maria Catargiu and Mircea Grigoras \\ "P. Poni” Institute of Macromolecular Chemistry, 41A Gr. Ghica Voda Alley, Iasi-700487, Romania \\ *Email: anca.bendrea@icmpp.ro, Phone: +40 232 21754, Fax: +40 232211299
}

\begin{abstract}
Mesoporous SBA-15/conducting polymers composites, type SBA- polithiophene, SBA-polyaniline, respectively were synthesized and their physical properties were investigated. Polymers molecules were synthesized by chemical oxidative polymerization inside the pores of the mesoporous material by a post-synthesis process. The obtained materials were characterized by X-ray diffraction, scanning electron microscopy, thermal analysis and infrared spectroscopy.
\end{abstract}

\section{Introduction}

Chemical sensors for gases are at the forefront of the information acquisition chain about the environment in which we live. The quality of the air is one of the most important concerns of our society. A large number of gas sensors use the organic components, like porphyrins, phtalocyanines, or doped conducting polymers [1, 2]. Conducting polymers, such as polypyrrole (PPy), polyaniline (PANi), polythiophene (PT) and their derivatives have been used as the active layers of gas sensors since early 1980s [3]. In comparison with most of the commercially available sensors, based on metal oxides and operated at high temperature, the sensors that have conducting polymers like component exhibit many improved characteristics. They have high sensitivities and short response time even at room temperature. This endows conducting polymers sensors with low energy consumption and simple device configuration. The selectivity of sensors can be easily modified by the adjustment of the specific properties of conducting polymers, by introducing different substituents, or copolymerizing with different monomers. However, conducting polymers have several disadvantages like: long-time instability and irreversibility, low selectivity that need to be improved.

To meet the need for analyzing the gas mixture and to overcome the low stability, poor selectivity and high cost problems of popular sensors, organic-inorganic hybrid composite sensors are intensively investigated [4-7]. They can complement the characteristics of pure inorganic and organic materials. It is also observed that hybrid materials have small grain size and high stability in air $[8,9]$.

In this work we exploit the hybrid organic-inorganic self-assembly approach to prepare mesostructured conducting polymer silica composite particles, type polyaniline/SBA-15 (SBA-PANi), polythiophene/SBA-15 (SBAPT) respectively. Mesoporous materials are of great interest because of their potential applications in various areas that span from catalysis to drug delivery [10-14].The uniform pore architecture of mesoporous materials make them very attractive as hosts for the encapsulation of polymers. In fact, several reports describe the encapsulation of conducting polymers such as polyppyrole, polythiophene and polyaniline into molecular sieves [15-21]. We focus on conjugated polythiophene and polyaniline, two of the most promising conducting polymers due to a good combination of properties, stability, price and ease of synthesis.

Our mesostructured conducting polymers silica composites particles were prepared via oxidative polymerrization of monomers by iron (III) chloride, in the case of thiophene and ammonium persulfate when aniline is the monomer, in the presence of SBA-15 particles. The obtained composites were characterized by X-ray diffraction, infrared spectroscopy, scanning electron microscopy, thermal analysis and. The preliminary results obtained for composite particles will be used in our further investigation regarding the development of humidity sensors.

\section{Results and discussion}

The powder XRD patterns for SBA-15, SBA-15-polythiophene and SBA-polyaniline composites are presented in Fig. 1. The successful formation of well-ordered mesoporous materials with two-dimensional hexagonal (p6mm) structure, with $d_{100}$ spacing of $9.1 \mathrm{~nm}$, follows from the presence of a basal (100) peak, along with (110) and (200) reflections in the low-angle region [22].

The XRD patterns of SBA - composite materials (Fig 1b, 1c) show that the composite materials are not as well ordered as the regular SBA-15, as evidence by the broadening of the (100) reflection peak and the disappearance of the higher order reflection. The XRD data show an increase in the $d_{100}$ spacing from $9.1 \mathrm{~nm}$ to $15.1 \mathrm{~nm}$ in the case of SBA -PT composite and to $12.1 \mathrm{~nm}$ for SBA- polyaniline samples. This enhancement of $d_{100}$ spacing is attributed to encapsulation of polymers into SBA-15 structures.

The presence of polymers molecules in the mesoporous silica was also confirmed by infrared spectroscopy (Fig. 2). The FT-IR spectrum of silica mesoporous SBA-15 (Fig. 2a) shows typical vibrations of SBA-15 silica framework with the asymmetric Si-O-Si stretch recorded at around $1080 \mathrm{~cm}^{-1}$. The symmetric stretch occurs at 809 $\mathrm{cm}^{-1}$, while the band at $460 \mathrm{~cm}^{-1}$ is assigned to the Si-O-Si bending mode [23]. 


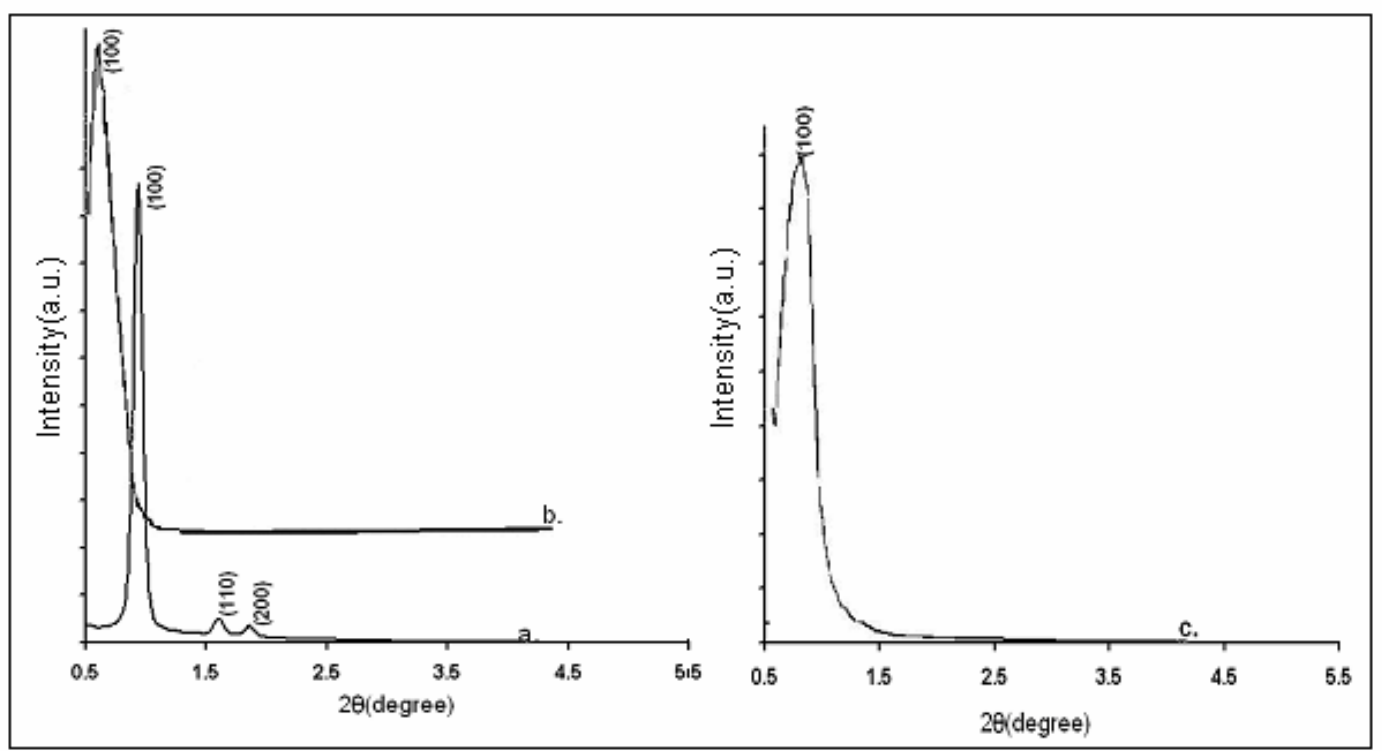

Fig.1. XRD patterns for a. SBA-15, b. SBA-PT and c. SBA-PANi composites

All these vibrations characteristics to silica SBA-15 are presented in the FT-IR spectra of SBA-PT and SBAPANi, respectively, composites (Fig.2b, 2c), but some of them are shifted due to the presence of polymer. Also, the polythiophene presence in the composite it is confirmed by the absorption peak at $848 \mathrm{~cm}^{-1}$ ascribed to the C-H outof -plane stretching vibration of the 2,5-substituted thiophene ring, which confirmed the polymerization of monomer[24]. The peak at $696 \mathrm{~cm}^{-1}$ can be assigned to the C-S bending mode, which also, indicates the presence of polymer in the composite structure.

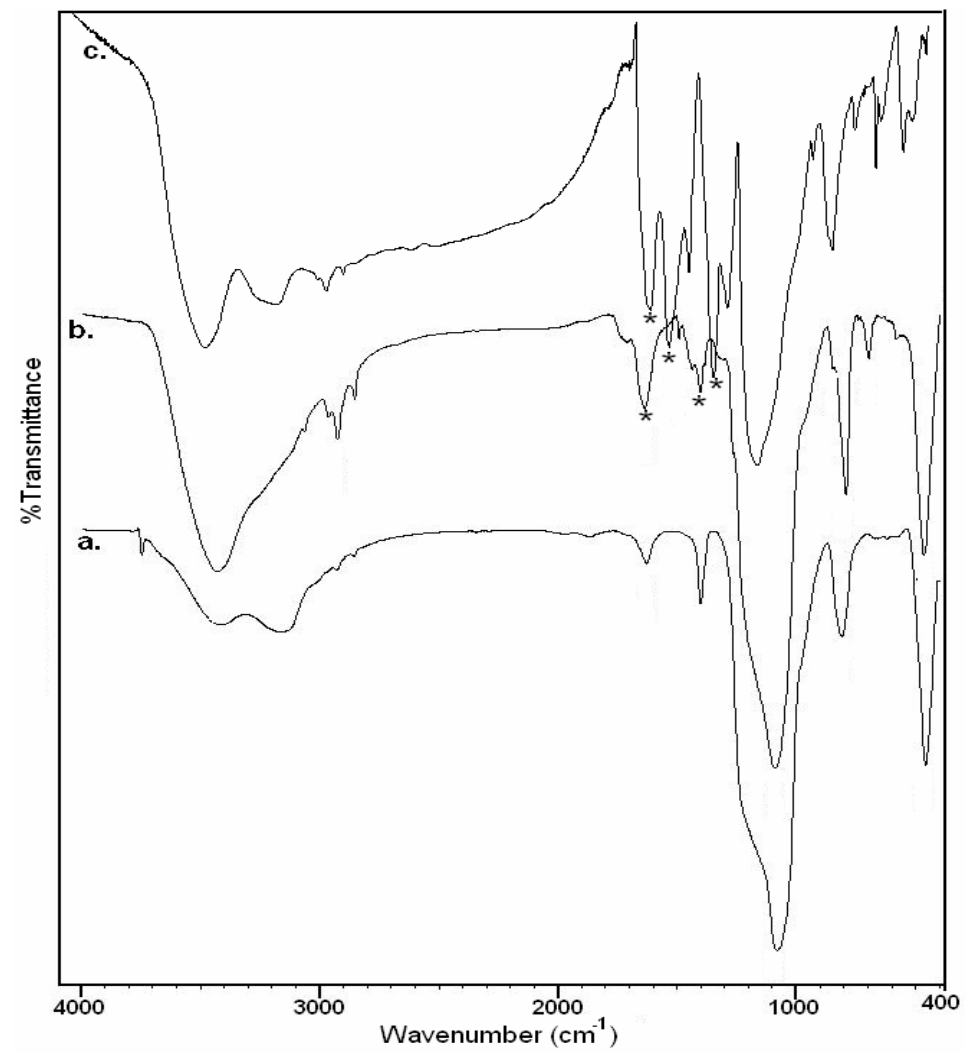

Fig. 2. FT-IR spectra of a.SBA-15, b. SBA-PT and c. SBA-PANi composite samples (* marks some of the polymers characteristics bands)

The FTIR spectra of SBA-PANi composite (Fig.2c) show both, typical vibrations of the SBA-15 silica framework and also display bands associated with PANi at 1564, 1486 and $1299 \mathrm{~cm}^{-1}$. These bands are associated 
with the stretching vibrations of the quinoid and benzenoid rings. The band at around $801 \mathrm{~cm}^{-1}$ is attributed to aromatic $\mathrm{C}-\mathrm{H}$ bending out of plane of the 1,4-sustituted aromatic rings [25,26].

Further evidence of the presence of polymers was confirmed by TGA analysis as shown Fig.3.
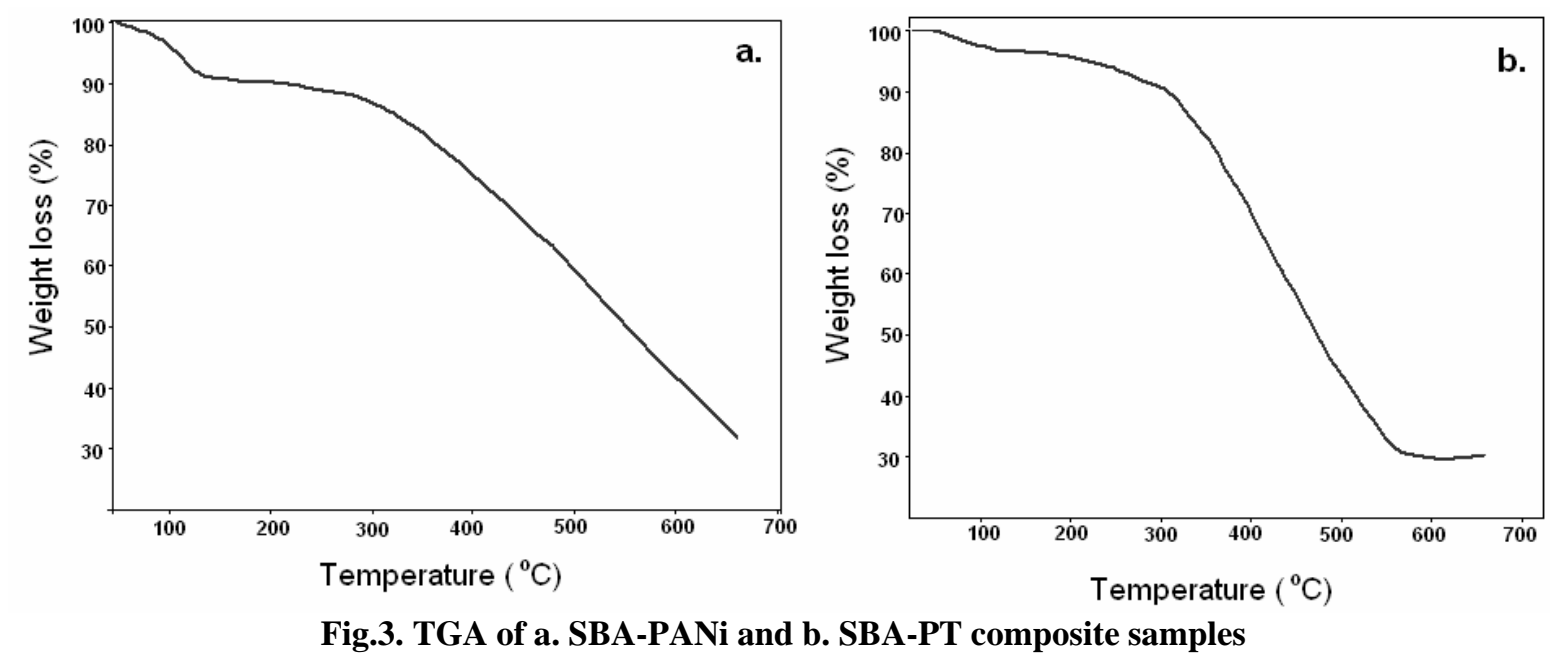

The trend of degradation of the SBA-PANi composites (Fig.3a) is similar to that of pure PANi and show two major step weight loss process. The first step indicates a $9.2 \%$ weight loss at temperatures up to $100^{\circ} \mathrm{C}$. This step can be attributed to the expulsion of water molecules and the dopant $(\mathrm{HCl})$ from PANi chain. The second-step weight loss occurs between 315 and $675^{\circ} \mathrm{C}$, which is due to the degradation of PANi chain. Note that the decomposition rate when the polymer is encapsulated is very different from the decomposition rate of bulk polymers [17].The decomposition of polymer backbone of pure PANi usually occurs around $300-450^{\circ} \mathrm{C}$, but the encapsulated polymer decomposes slowly from about 315 to $675^{\circ} \mathrm{C}$.

The thermogravimetric curve of SBA-PT composites (Fig.3b) also reveals two weight-loss steps. The first one, up to $300^{\circ} \mathrm{C}$ indicates a $5 \%$ weight loss is attributed to the evaporation of adsorbed water molecules on the sample surface and to the expulsion of oligomers. The most important weight loss (about $38 \%$ ) takes place between 300 and $650^{\circ} \mathrm{C}$. Above $650^{\circ} \mathrm{C}$ the organic component is completely decomposed, so the weight loss recorded in this step can be attributed to the inorganic silica.

The minimum temperature in the case of our composites is shifted to a higher temperature suggesting an increase of thermal stability of SBA-polymers composites in comparison with pure polymers, which proved that the encapsulation of polymers into mesoporous silica enhances their thermal stability.

Thermogravimetry was also employed to determine the amount of polymer in the composite samples. It was found that the composites contained $65 \%$ of PANi and $45 \%$ of PT, respectively. The amount of polymer in the composite was calculated from the mass loss between $300^{\circ} \mathrm{C}$, when the solvent was evaporated and $700^{\circ} \mathrm{C}$, when the polymer was completely decomposed.

Fig. 4 shows the particle morphology of both SBA-15 and SBA-PT composites observed by scanning electron microscopy (SEM).
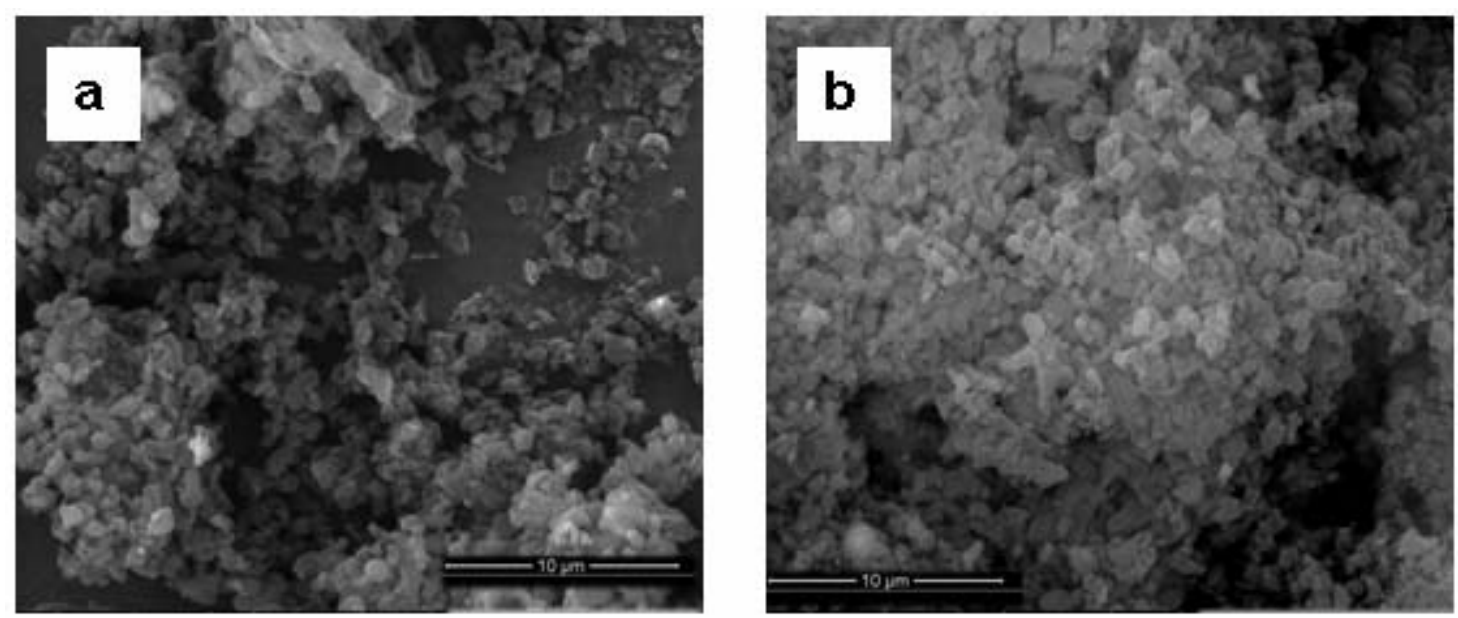

Fig.4. SEM images of a. SBA-15 and b. SBA-PT composites 
The SEM images (Fig. 4) reveal that the mesoporous materials are assemblies of many doughnut-like particles being typical of SBA-15 materials [27]. As shown from SEM images, no apparent difference in the particle surface morphology between the SBA-15 and SBA-PT composites (Fig.4b), except the fact that composite structure is more compact than SBA-15. This agglomeration effect is attributed to the presence of polythiophene on composite structure.

\section{Experimental}

Tetraethylorthosilicate (TEOS) (98\%), hydrochloric acid (37\%), fresh distilled thiophene, aniline and ammonium persulfate were purchased from Aldrich. The triblock copolymer Pluronic 123 (P123) was supplied by BASF. Water-free iron (III) chloride, was purchased from Fluka. All chemicals were used as received without any further purification.

\section{Materials Preparation}

Silica SBA-15 was synthesized following the literature procedure [22, 26]. $4 \mathrm{~g}$ of ploy(ethylene oxide)poly(propylene oxide) - poly(ethylene oxide) triblock copolymer $\left(\mathrm{PEO}_{20}-\mathrm{PPO}_{70}-\mathrm{PEO}_{20}\right)$, Pluronic 123 , were added to $150 \mathrm{ml}$ of $1.6 \mathrm{M}$ aqueous solution of hydrochloric acid and stirred for 16 hours. Next, $8.5 \mathrm{~g}$ TEOS were added dropwise and the polymer - TEOS synthesis mixture was stirred for 24 hours. The synthesis gel was transferred to Teflon-lined sealed containers and kept at $100^{\circ} \mathrm{C}$ for 48 hours under static conditions. The final product was filtered, washed with water and dried at $80^{\circ} \mathrm{C}$ over night. The surfactant, Pluronic P123, was removed by a heat treatment in air, at $550^{\circ} \mathrm{C}$, for 6 hours.

Preparation of SBA-PT composites. $0.1 \mathrm{~g}$ of SBA-15 was dried under vacuum at around $100^{\circ} \mathrm{C}$ for 24 hours and then placed in $10 \mathrm{ml}$ chloroform (water free) and stirred at room temperature for 2 hours. Next, $0.2 \mathrm{ml}$ thiophene was added to this mixture and stirred at room temperature for another 24 hours. Meanwhile, the dispersion of $0.304 \mathrm{~g}$ $\mathrm{FeCl}_{3}$ in $10 \mathrm{ml} \mathrm{CHCl}_{3}$ was prepared and subsequently added to the dispersion of SBA-monomer solution. After 24 hours of vigorous magnetic stirring at room temperature, the composite was extracted with methanol, filtered, washed with deionized water and $2 \mathrm{M} \mathrm{HCl}$ aqueous solution and dried at $60-80^{\circ} \mathrm{C}$ at vacuum. During the extraction step, the color of the composite changed from black (PT in oxidized state) to red (PT in reduced state), indicating successful reduction of the polymer.

Preparation of SBA-PANi composites. $0.1 \mathrm{~g}$ of SBA-15 was dried under vacuum at around $100^{\circ} \mathrm{C}$ for 24 hours and then placed in $10 \mathrm{ml}$ of $1 \mathrm{M} \mathrm{HCl}$ aqueous solution and stirred at room temperature for 2 hours. Next, $0.2 \mathrm{ml}$

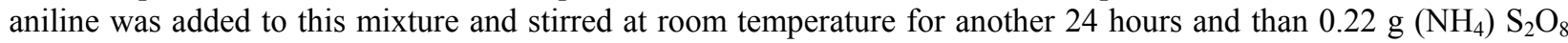
were added to the reaction system. After 24 hours of vigorous magnetic stirring at room temperature, the composite was extracted with methanol, filtered, washed with deionized water and $2 \mathrm{M} \mathrm{HCl}$ aqueous solution and dried at 60 $80^{\circ} \mathrm{C}$ at vacuum. During the extraction step, the color of the composite changed from blue (PANi in oxidized state) to dark green (PANi in reduced state), indicating successful reduction of the polymer.

\section{Materials Characterization}

Powder X-ray diffraction patterns were obtained with a Brucker AD8 ADVANCE X-ray diffractometer using $\mathrm{CuK} \alpha$ radiation. The FTIR spectra (KBr pellets) of samples were recorded in the range of $4000-400 \mathrm{~cm}^{-1}$, using a Bruker Vertex 70 spectrometer. The SEM studies were performed on samples fixed by means of colloidal silver on copper supports. The samples were covered with a thin layer of gold, by sputtering (Emitech K550X). The coated surface was examined by using an Environmental Scanning Electron Microscope (ESEM) Quanta 200-type, operating at $15 \mathrm{kV}$ with secondary electrons. Thermal gravimetric analysis (TGA) was performed by means of a Mettler Toledo TGA-SDTA 851 device, in air stream, a heating speed of $10 \mathrm{~K} / \mathrm{min}\left(25-800{ }^{0} \mathrm{C}\right.$ range $)$ and the sample weight of 4-6 mg. Constant operating parameters were kept for all the samples in order to obtain comparable data.

\section{Conclusions}

This study described the preparation of new organic-inorganic hybrid composites, where organic component is a conducting polymer, type polyaniline, or polythiophene and mesoporous silica SBA-15 represent the inorganic host. In situ chemical oxidative polymerization method of the monomers has been used for the composites synthesis.

The dates obtained from XRD and FTIR analysis show the formation of polymers and the successful encapsulation of them into SBA-15 structure. The morphology of SBA-PT samples was obtained by SEM. By thermogravimetric analysis was reveals the main weight loss and decomposes steps of composites. Thermogravimetry was also employed to determine the amount of polyaniline and polythiophene, respectively, in the composites.

These preliminary results obtained for mesoporous silica-conducting polymers composites will be used in our further investigation regarding the development of humidity sensors.

\section{Acknowledgments}

The authors thank the Romanian National Authority for Scientific Research for financial support (Grant PN II-IDEI-963). 


\section{References}

[1]. McQuade,D. T., Pullen,A. E.\& Swager, T. M. Chem. Rev.2000, 100, 2537-2574.

[2]. Gerard, M., Chaubey,A.\& Malhotra, B. D. Biosens. Bioelectron. 2002, 17, 345-359.

[3]. Bai, H. and Shi, G., Sensors, 2007, 7, 267-307.

[4]. Guernion, N., B.P.J. de Lacy Costello, Ratcliffe, N.M., Synth. Met.2002, 128,139

[5]. Tandon, R.P., Tripathy, M.R., Arora, A.K., Hotchandani, S., Sens. Actuators B 2006, 114,768-773.

[6]. Ram, M.K., Yavuz, O., Aldissi M., Synth. Met. 2005, 151, 77-84.

[7]. Sadek, A.Z., Wlodarski, W., Shin, K., Kaner, Kalantar-Zadeh, R.B. K., Nanotechnology. 2006, 17, 4488-4492.

[8]. Parch, R., Gangolli, S.G., Matijevic, E., J. Colloid Interf. Sci.1991, 144 (1), 27-35.

[9]. Maeda, S., Armes, S.P., Chem. Mater.1995, 7, 171-178.

[10]. Kresge C.T., Lenowicz M.E., Roth W.J., Vartuli J.C., Beck J.S., Nature,1992, 359, 710.

[11]. Diaz J.F., Balkus Jr. K.J., J. Mol. Catal. B: Enzym.1996, 2,115.

[12]. Jain T.K., Roy I., De T.K., Maitra A., J. Am. Chem. Soc.1998, 120, 11092.

[13]. Ying J.Y., Mehnert C.P., Wong M.S., Angew. Chem. Int. Ed. 1999, 38, 56.

[14]. Vallet-Regi M., Ramila A., del Real R.P., Perez-Pariente J., Chem. Mater.2001, 13, 308.

[15]. Mbaraka I.K., Radu D.R., Lin V.S.-Y., Shanks B.H., J. Catal.2003, 219, 329.

[16]. Wu C.-G., Bein T., Science. 1994, 264, 1757.

[17]. Enzel P., Bein T., J. Phys. Chem.1989, 93, 6270.

[18]. Cho M.S., Choi H.J., Ahn W.-S., Langmuir.2004, 20, 202.

[19]. Cho M.S., Choi H.J., Ahn W.-S., Synth. Met.2003, 711, 135-136.

[20]. Cho M.S., Choi H.J., Kim K.Y., Ahn W.-S., Macromol. Rapid Commun.2007, 23, 713.

[21]. Cardin D.J., Adv. Mater. 2002, 14, 553.

[22]. Zhao D., Feng J., Huo Q., Melosh N., Fredrickson G.H., Chmelka B.F., Stucky G.D., Science, 1998, $279,548$.

[23]. Coutinho D., Yang Z., Ferraris J.P., Balkus K.J., J. Micropor.Mesopor.Mater, 2005, 81, 321-332.

[24]. Han M.G., Foulger S.H., Adv. Mater, 2004, 13(4), 301.

[25]. Long, Y., Chena, Z., Duvailb, J.L., Zhang, Z. and Wan, M. Physica B.2005, 370: 121-130.

[26]. Li, P., Yu, B. and Wei, X. J. Appl. Polym. Sci.2004, 93: 894-900.

[27]. Zhao D., Sun J., Li Q., Stucky G. D., Chem. Mater, 2000, 12, 275-279. 